\title{
Theory Summary
}

\section{Larry McLerran*}

Institute for Nuclear Theory

University of Washington Seattle, WA 98195

E-mail: lmcler@uw.edu

I provide a short summary of many of the theoretical talks given at "The Critical Point and the Onset of Dconfinement Conference", "CPOD 2018", held in Corfu, Greece, September, 2018. I also include some remarks on a possible interpretation of the recent observation that the sound velocity squared may be of the order or exceed $1 / 3$ at a few times nuclear matter density, as determined by neutron star observations.

Corfu Summer Institute 2018 "School and Workshops on Elementary Particle Physics and Gravity" (CORFU2018)

31 August - 28 September, 2018

Corfu, Greece

${ }^{*}$ Speaker. 


\section{Introduction}

During this scientific meeting, you have been shown many times "The Phase Diagram of QCD". Problem is you were shown many different phase diagrams! There is a reason for this. Outside of a small region around zero baryon density, we do not know the phase diagram. The region around small baryon density and finite temperature has been well studied by lattice gauge theory computations that provide reliable results at such small baryon density, and by many experimental results of the highest quality from both the LHC and RHIC. Non-perturbative computations at finite baryon density on the lattice are unreliable because there is no convergent method to provide convergent results. Simulations of the properties of lower energy nuclear collisions are rapidly improving, but much remains to be done both in experiment and theory to develop reliable and compelling understanding of high density matter. In a somewhat surprising development, data from neutron star observations now provide the best experimental constraints on the equation of state for zero temperature finite density strongly interacting matter.

It is of course great fun to be an experimentalist or a theorist exploring an area where little is known. Oftentimes, in such conditions, wrong ideas became widely and strongly held, simply because there is no compelling way to refute them. We must remember that we are dealing with speculative science, and I strongly recommend the famous quote by Mark Twain to you all:

It is not what you know that gets you in trouble, but what you think you know but you do not.

An interesting result that has come from analysis of experimental data are the decoupling curves where final state multiplicities of various flavors of particles are used to infer a decoupling temperature and chemical potential. See Ref. [1] for reference to original material. See the presentation of Galatyuk[2]. (References are to conference presentations unless otherwise noted.) A result of such a determination is shown in Fig. 1. I have drawn a blue line and a red line on the figure. The blue line would be the case if the de-confinement cross-over transition occurred at a fixed temperature and the straight red line would be the case for

$$
\left(M-\mu_{B}\right) / T=\text { cons }
$$

Such behaviour is suggested by large $N_{c}$ arguments[3]. While one can seriously question the decoupling interpretation of these curves, at least at high baryon density and low temperature, it is clear that something changes at baryon chemical potential of about $400 \mathrm{MeV}$. This simple observation provides a partial justification of the excitement about the low energy run as an attempt to understand the properties of matter at finite density, and that this matter may have different properties from that of high temperature and low baryon density matter. The point at which the two straight lines intersect is approximately the point at which baryons dominate the energy density of strongly interacting matter.

We were also presented with data from NA61 by Grabieskow[4] that showed in the region of this transition that various ratios of particle abundances show distinctive changes in their dependence on collision energy, and that there are intriguing structures associated with intermittancy. The first is associated with the so-called Horn originally observed by Gazdzicki[5]. Some computations using three flavor NJL models were shown to reproduce such behavior by Freisen[6]. 


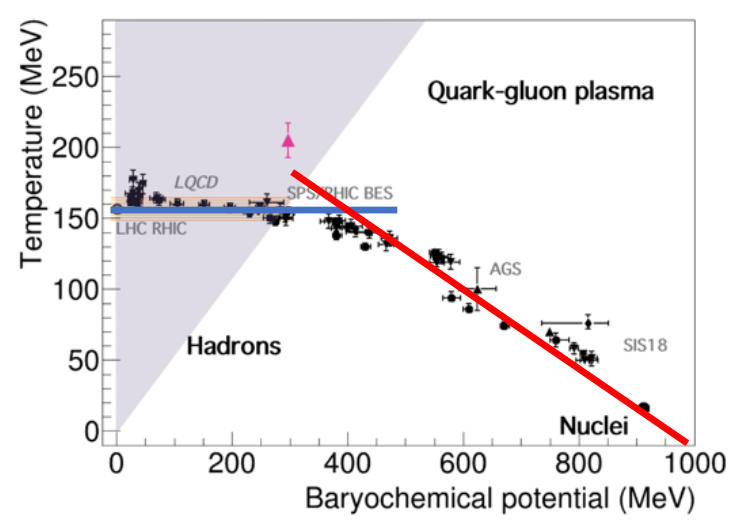

Figure 1: Data on decoupling temperature and density.

In seminal work by Antoniou and Diakonos[7] it was argued that intermittency might be a signal for a critical end point. They used a sigma model to simulate the approach to criticality. The approach to masslessness of the sigma field in such a theory generates scale invariant correlation functions that might be responsible for the observed effect in NA61[4]. The effect is localized in energy, A, and collision centrality since one has to be very near to critical point to get a sizable signal. How does this emerge in collisions at neighboring centrality, energy and A? If it is a real effect it should have a maximum somewhere and it should be be possible to follow in the multidimensional space of energy, impact parameter and A.

The masslessness of a sigma field mode might generate long range attractive forces and induce large scale baryon number fluctuations as argued by Torres-Rincon[8]. This might generate clustering effects in baryons. Such effects should persist below the critical end point since first order phase transitions have large fluctuations in baryon number.

As argued a long time ago by Stoecker[9], it is in this region that various hydrodynamic flow signatures, in particular directed flow, show non-monotonic properties.

\section{Proton Number Fluctuations}

In the talk of Galatyuk[2], various measures of proton number flucutations were presented. Baryon number fluctuations and correlations are among the strongest potential signatures for phase transitions since if the transition is related to chiral symmetry breaking, the nucleon mass might be strongly affected during such a transition. Many times, experimentalists and theorists have looked for signatures in two particle baryon number correlations without success. It was however recently argued by Bzdak, Koch and Skokov[10], that while 2 particle correlations give small deviation from statistical fluctuation, two particle correlations give a moderate effect and three 4 particle correlations a very large effect. The 2, 3 and for particle correlations as a function of energy are shown for the Star experiment in Fig. 2, and for the Hades experiment for the fluctuations as a function of hadron multiplicity, Fig, 3.

While it is tempting to speculate that the correlation seen in these experiments might be tied to a phase transition, detailed computations such as those of Bzdak, Koch and Skokov[10] cannot 


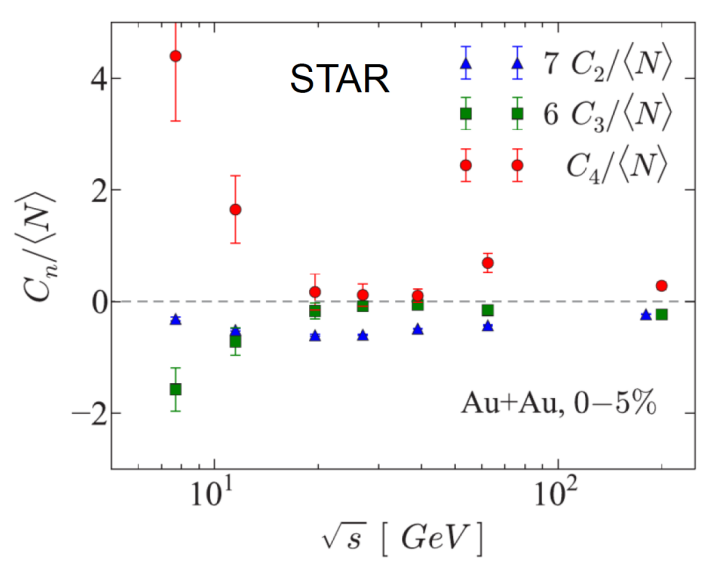

Figure 2: Multiparticle proton correlations as a function of energy in the STAR experiment[2].

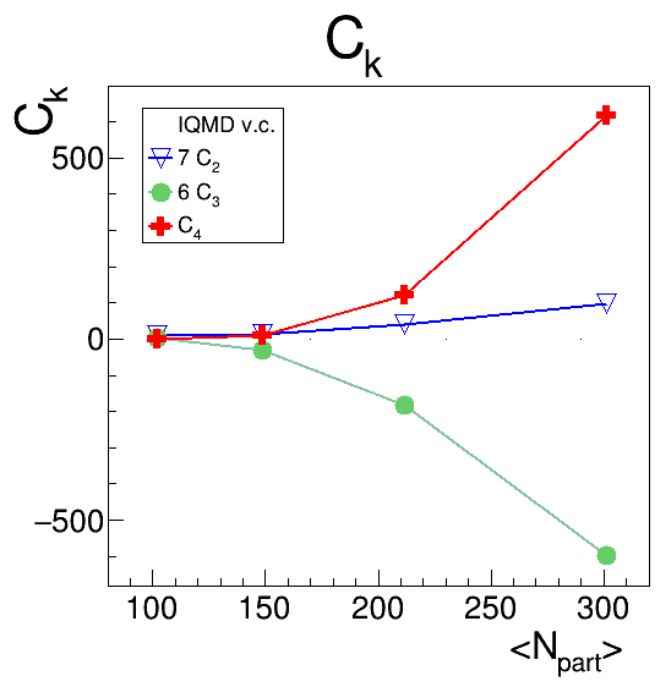

Figure 3: Multiparticle proton correlations as a function of multiplicty in the Hades experiment[2].

succesfully describe these correlations by a model with such a transtiion as an underlying property. It appears that one needs two distinctive event classes, each with a Poisson distribution centered around a different value. This is difficult to resolve with an underlying theory where for example one had a first order phase transition since in such a circumstance one would produce a mixed phase, and the underlying fluctuation spectrum would be a product of two Poisson distributions which is itself Poisson, and not a sum of two Poisson distributions, While the data is suggestive and provocative, a fundamenal understanding is at this time lacking.

\section{Thermalization and Critical Behaviour}

The classic work developed by Braun-Munzinger, Redlich and Stachel[11],[12], demsonstrates that to a very good approximation, the ratios of abundances of particles produced in heavy 
ion collisions is described as a thermal distribution with a baryon number chemical potential. The temperature and chemical potential is a function of multiplicity and energy. This result is interpreted as that the system quckly freezes out at some temperature and density close to the line of crossover in the $\mu_{B}$ and $T$ plane. This can be strongly motivated at low baryon density, but such an interpretation is controversial at high baryon density.

At high energies, there is some tension between optimizing thermal fits for strange and nonstrange baryons. Noronha-Hostler argued that there may be a different freeze out temperature for strange vs non-strange baryons[13].

The particle abundance curve also works well in small systems where the assumption of chemical equlibrium might be questioned. In the talk of Bellwied it was argued that perhaps this thermal spectrum might be a consequence of quantum entanglement, and not due to thermalization as in the conventional way of thinking[14].

In the talk of Rennecke the effects of stangeness neutrality were considered for various fluctuation measures[15].

The position of the cross over transition as a function of baryon number density can be quantitatively explored using lattice gauge theory methods up to a baryon number chemical potential of order $250 \mathrm{MeV}$ [16]. The position of this crossover is sem-iquantitatively simliar to the values of $\mu_{B}$ and $T$ measured by particle abundances. In analysis presented by Karsch, Mukherjee, Goswami and Vovchenko, computations were shown that also argued that there is no evidence for a critical endpoint for temperatures below $300 \mathrm{MeV.[17],[18],[19],[20].} \mathrm{The} \mathrm{dependence} \mathrm{of} \mathrm{the}$ crossover temperature on chemical potential is very weak in this region, consistent with large $N_{c}$ expectations.

Glozman reminded us that there is no confinement phase transition nor even sharp cross over. He asks how does one conceptualize the degrees of freedom on either side of the cross over? Perhaps Euclidean correlation functions are sensitive to confining effects even beyond the crossover[21]?

The crossover transition seen in lattice gauge theory computations is a remant of a chiral phase transition in the limit that the light quarks masses go to zero. In a true phase transition, there would be a singularity along a line of in the $\mu_{B}, T$ plane. This singularity would appear in the derivatives of various measurable quantities, and these derivatives correspond to operators that measure fluctuations of various quantities such as baryon number density or energy density. For finite quark masses there is no true singulrtity, but sharp behaviour. This work has been most strongly developed by Karsch and Redlich[17].

In a number of presentations by Herold, Nahrgang, Wink, Bluhm, Yin, Ridgway, Sochorova, Tomasik and Rustamo, it was shown how such underlying behaviour might be measured and computed in heavy ion collisions[22],[23],[24],[25],[26],[27],[28] [29],[30]. In particular, one needs to understand for various initial conditions how close does one evolve to a hypothetical critical end point and how does such an evolution affect the extraction of underlying fluctuation measures.

\section{The Chiral Magnetic and Chiral Vortical Effect}

Heavy ion collisions allow one to probe matter in the presence of high magnetic fields and at high angular momentum.. Such magnetic fields and angular momenta may generate topological 
excitations of the underlying field theory. For example, if there is a fluctuation in the underlying helicity of quarks, then an external magnetic field will generate an electromagnetic current. This is the Chiral Magnetic Effect[31]. High angular momentum can generate vortices[32]. Mao discusssed the general problem of QCD in external magnetic fields[33]. Sorin discussed the chiiral magnetic and chiral vortical effect, and presented beautiful simulations of velocity and vortex fields that might be produced in collisions at NICA energies[34].

\section{Varying the Baryon Number Density at High Energy}

Most of the discussion of high baryon number density concerns collisions at low energy. Some thought needs to be given to the proposal described by Brewe[35]: Are we better to change baryon density by varying rapidity at fixed energy or by varying the energy over a fixed region of rapidity? In principal one could study the high baryon density region by going to very high energy and looking in the target fragmentation region. There are arguments that $T / \mu_{B}$ is approximately beam energy independent at fixed rapidity. This means that one can start with very high $\mathrm{T}$ and $\mu_{B}$ and it evolves with this roughly fixed rapidity[36]. After expansion, one has produced a baryon rich system like that produced at low energy, except that it is expanding very slowly. To properly set up this problem, one needs a good theoretical treatment of initial conditions.

\section{Neutron Stars}

The recent measurments of neutron star properties such as mass radii and gravitational radiation from neutron star collisions has led to a better undertsanding of the equation of state of high baryon density matter. As argued by Stoecke, shown in Fig. 4, neutron star collsiions occur over a range of temperature and density that might be accessable to laboratory experiment

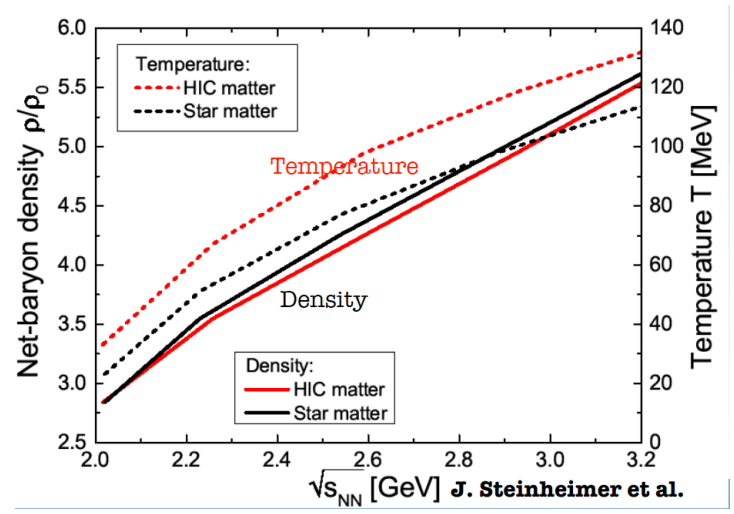

Figure 4: Temperature and density accessable in lab experiment sand probed in neutron star collisions

Constraints from neutron star mass radius relations have led to a very interesting constraint on equation of state of nuclear matter. For a recent article on such deternination and references to the original literature, see Ref. [37]. At energy densities only a few times nuclear matter densities, these analyses agree that the sound velocity squared should become (in units of the speed of light)

$$
v_{s}^{2} \geq 1 / 3
$$


This is a truly remarkable result, The sound velocity for a zero temerpature gas is determined by

$$
v_{s}^{2}=\frac{N_{B}}{\mu_{B} d N_{b} / d \mu_{B}}
$$

This can be reexpressed as

$$
\frac{\delta \mu_{B}}{\mu_{B}}=v_{s}^{2} \frac{\delta N_{B}}{N_{B}}
$$

In this equation, the baryon chmical potential or Fermi energy includes the effect of the nucleon mass. If the sound velocity is of order one, then an order one change in the baryon density indeuces a change in the Fermi energy of nucleon that is of the order of the nucleon mass! This is far different from the situation of nuclear matter where the Fermi energy (minus the nucleon mass) is of the order of 10's to at most $100 \mathrm{MeV}$.

While it may be true that even at such high chemcal potential, the energy density of nuclear matter might still be computed using conventional nuclear many body theory, the assumptions of such computations are surely open to doubt. In presentations of Blaschke, Motornenko and Fischer, various quark model computations were described[38], [39], [40].

We can easily see that understanding a relativistic sound veloicty for nuclear matter is not so easy. If would be tempting to say it was due to chiral symmetry restoration. The Fermi momentum of a nucleon is $k_{F}=\sqrt{\mu_{B}^{2}-M_{B}^{2}}$. If $M_{B}$ shrinks to zero as the chemical potnential increases, then at zero $M_{B}$, the Fermi momentum will be $k_{F}^{B} \geq M_{B}$. This corresponds to a huge baryon density $\rho_{B} \sim k_{F}^{3} \sim M_{B}^{3}$ that is about 100 times larger than that of nuclear matter. This is too high a density to be relevant for neutron stars.

Tho avoid this problem we can introduce quark degrees of freedom[41]. In this case if we make a transition between a system dominated by nucleons to one dominated by quarks, effectively the baryon chemical potential, which at nuclear matter density corresponds to a Fermi momentum of order $k_{F}^{B} \sim 300 \mathrm{MeV}$, can become of order $k_{F}^{B} \sim M_{B}$. However if during this transition, the degrees of freedom are entirely transferred into quarks, the density does not go up so high, because $k_{F}^{Q} \sim k_{F}^{B} / 3 \rightarrow M_{B} / 3 \sim 300 \mathrm{MeV}$. Therefore the baryon density in quarks does not increase by a huge amount.

This feature of QCD can be uderstood in the large $N_{c}$ limit and the ability to transform continuously nucleon degrees of freedom into quark degrees of freedom is a characteristic of Quarkyonic Matter[3].

Such a transition means that the baryon chemical potential may increase by a large amount without the baryon number density much changing. The pressure therefore goes up rapidly. The energy density (that is dominated by the nucleon mass times the density) also does not rapidly change. The transition to quarks in Quarkyonic Matter does not involve a phase transition. It is quite the opposite. In the large $N_{c}$ limit, the baryon chemical potential and pressure jumps by a huge amount at almost constant energy density and baryon number density. This is the precise opposite of what occurs for a phase transition. This is precisely what is needed to generate the hard equation of state needed to support neutron stars. 


\section{Acknowledgements}

. The work of L. M. . was supported by the U.S. DOE under Grant No. DE-FG02-00ER41132.

\section{References}

[1] A. Andronic et al., Nucl. Phys. A 837 (2010) 65 doi:10.1016/j.nuclphysa.2010.02.005 [arXiv:0911.4806 [hep-ph]].

[2] T. Galatyuk : "Decoding the Phase structure of QCD at high IijB with HADES"

[3] L. McLerran and R. D. Pisarski, Nucl. Phys. A 796 (2007) 83 doi:10.1016/j.nuclphysa.2007.08.013 [arXiv:0706.2191 [hep-ph]].

[4] K. Grebieszkow : "New results on spectra and fluctuations from NA61"

[5] M. Gazdzicki and M. I. Gorenstein, Acta Phys. Polon. B 30 (1999) 2705 [hep-ph/9803462].

[6] A. Friesen : "Strange matter and kaon to pion ratio in SU(3) PNJL model"

[7] F. Diakonos : "Finite-size scaling, Intermittency and the QCD critical point"

[8] J. Torres-Rincon : "Nuclear correlations and modifications of the nucleon-nucleon potential due to the QCD critical mode"

[9] S. A. Bass et al., Prog. Part. Nucl. Phys. 41 (1998) 255 [Prog. Part. Nucl. Phys. 41 (1998) 225] doi:10.1016/S0146-6410(98)00058-1 [nucl-th/9803035].

[10] A. Bzdak, V. Koch and V. Skokov, Phys. Rev. C 87 (2013) no.1, 014901 doi:10.1103/PhysRevC.87.014901 [arXiv:1203.4529 [hep-ph]].

[11] P. Braun-Munzinger, I. Heppe and J. Stachel, Phys. Lett. B 465 (1999) 15 doi:10.1016/S0370-2693(99)01076-X [nucl-th/9903010].

[12] P. Braun-Munzinger, K. Redlich and J. Stachel, "Particle production in heavy ion collisions," In *Hwa, R.C. (ed.) et al.: Quark gluon plasma* 491-599 [nucl-th/0304013].

[13] J. Noronha-Hostler : "Freeze-out temperature from net-Kaon fluctuations at RHIC"

[14] R. Bellwied : "Composite particle production in relativistic particle collisions through quantum entanglement"

[15] F. Rennecke : "Strangeness Neutrality, Baryon-Strangeness Correlations and the Phase Structure of QCD"

[16] C. Ratti : "Lattice-based Equation of State of QCD matter with a critical point"

[17] Fr. Karsch : "News on net-charge fluctuations and correlations from lattice QCD"

[18] Sw. Mukherjee : "QCD phase diagram from lattice"

[19] J. Goswami : "Critical end points in (2+1)-flavor QCD with imaginary chemical potential"

[20] V. Vovchenko : "QCD equation of state at finite baryon density with fugacity expansion"

[21] L. Glozman : "Implications of the chiralspin symmetry for QCD at high temperatures and densities"

[22] Ch. Herold : "Production of Entropy at the Chiral Phase Transition from Dissipation and Noise"

[23] M. Nahrgang : "Dynamics of net-baryon density fluctuations near the QCD critical point" 
[24] N. Wink : "From first principle QCD to dynamical fluctuations"

[25] M. Bluhm : "Finite size effects on critical fluctuations"

[26] Y. Yin : "QCD critical point, fluctuations and hydrodynamics"

[27] G. Ridgway : "Understanding the out-of-equilibrium dynamics near a critical point with Hydro+

[28] R. Sochorova : "Evolution of multiplicity fluctuations in heavy ion collisions"

[29] B. Tomasik : "Benchmark values for net proton number fluctuations"

[30] A. Rustamov : "Identified Particle Fluctuations from ALICE at the CERN LHC"

[31] D. E. Kharzeev, L. D. McLerran and H. J. Warringa, Nucl. Phys. A 803 (2008) 227 doi:10.1016/j.nuclphysa.2008.02.298 [arXiv:0711.0950 [hep-ph]].

[32] D. E. Kharzeev and D. T. Son, Phys. Rev. Lett. 106 (2011) 062301 doi:10.1103/PhysRevLett.106.062301 [arXiv:1010.0038 [hep-ph]].

[33] S. Mao : "Chiral symmetry restoration and quark deconfinement beyond mean field in a magnetized PNJL model"

[34] Al. Sorin : "Vorticity, hydrodynamic helicity and polarization in baryon-rich matter"

[35] J. Brewer : "Searching for the QCD critical point via the rapidity dependence of cumulants"

[36] L. McLerran, EPJ Web Conf. 172 (2018) 03003. doi:10.1051/epjconf/201817203003

[37] Y. Fujimoto, K. Fukushima and K. Murase, arXiv:1903.03400 [nucl-th].

[38] D. Blaschke : "Chiral symmetry restoration by parity doubling and the structure of neutron stars"

[39] A. Motornenko : "Equation of state for hot QCD and compact stars from mean field approach"

[40] T. Fischer : "Explosions of massive blue-supergiant stars triggered by the QCD phase transition

[41] L. McLerran and S. Reddy, Phys. Rev. Lett. 122 (2019) no.12, 122701 doi:10.1103/PhysRevLett.122.122701 [arXiv:1811.12503 [nucl-th]]. 